\title{
Impact of Adaptive Radiotherapy on Locally Advanced Head and Neck Cancer - A Dosimetric and Volumetric Study
}

\author{
Abhinav Dewan ${ }^{1}$,SK Sharma ${ }^{1}$,AK.Dewan ${ }^{2}$, Himanshu Srivastava ${ }^{1}$, Sheh Rawat ${ }^{1}$, \\ Anjali Kakria ${ }^{1}$, Maninder Mishra ${ }^{3}$, Suresh $T^{3}$, Krati Mehrotra ${ }^{4}$
}

\begin{abstract}
Objective of the study is to evaluate volumetric and dosimetric alterations taking place during radiotherapy for locally advanced head and neck cancer (LAHNC) and to assess benefit of replanning in them. Materials and Methods: Thirty patients with LAHNC fulfilling the inclusion and exclusion criteria were enrolled in a prospective study. Planning scans were acquired both pre-treatment and after 20 fractions (mid-course) of radiotherapy. Single plan (OPLAN) based on initial CT scan was generated and executed for entire treatment course. Beam configuration of OPLAN was applied to anatomy of interim scan and a hybrid plan (HPLAN30) was generated. Adaptive replanning (RPLAN30) for remaining fractions was done and dose distribution with and without replanning compared for remaining fractions. Results: Substantial shrinkage of target volume (TV) and parotids after 4 weeks of radiotherapy was reported $(\mathbf{p}<0.05)$. No significant difference between planned and delivered doses was seen for remaining fractions. Hybrid plans showed increase in delivered dose to spinal cord and parotids for remaining fractions. Interim replanning improved homogeneity of treatment plan and significantly reduced doses to cord (Dmax, D2\% and D1\%) and ipsilateral parotid (D33\%, D50\% and D66\%) $(\mathbf{p}<0.05)$. Conclusions: Use of one or two mid-treatment CT scans and replanning provides greater normal tissue sparing alongwith improved TV coverage
\end{abstract}

Keywords: Adaptive radiotherapy - dosimetric analysis - anatomical changes - locally advanced head and neck cancer

Asian Pac J Cancer Prev, 17 (3), 985-992

\section{Introduction}

Radiotherapy alongwith chemotherapy plays a central role in treatment of locally advanced head and neck cancers (LAHNC) (Bhandari et al., 2014; Brouwer et al., 2015). Intensity modulated radiotherapy (IMRT) is presently considered a standard treatment for head and neck cancers (HNC) due to its ability to create highly conformal dose distribution with potential sparing of critical structures (Bhandari et al., 2014; Brouwer et al., 2015; Nath et al., 2009).

Planning target volume (PTV) drawn during CT based contouring and planning procedure accounts for organ motion and set-up uncertainties. However, this margin does not take into account anatomical alterations occurring due to weight loss, tumor shrinkage, change in muscle/fat mass and tissue edema during radiotherapy (Barker et al., 2004; Bhinde et al., 2010).

Sharp dose gradients between TV and OARs in IMRT result in drastic dosimetric changes. Therefore, small error in patient or tumor position/anatomy may have huge dosimetric and clinical impact (Hansen et al., 2006).
IMRT plan based on initial single CT scan acquired prior to radiotherapy may lead to unexpected complications and/or marginal recurrence if these uncertainties are not taken care off. Adaptive radiotherapy (ART) is a possible strategy to overcome these limitations and involves repeat imaging and replanning to adapt to actual patient anatomy (Castadot et al., 2010).

There is limited published data on ART in LAHNC in India and evidence of its usefulness may further help reduce treatment related morbidity. Purpose of the present study is to study and evaluate volumetric and dosimetric changes occurring during IMRT for LAHNC and to estimate benefit of replanning in them.

\section{Materials and Methods}

Between January'2012 to December'2014, thirty patients with LAHNC, fulfilling the inclusion criteria were enrolled in a prospective study conducted at Rajiv Gandhi Cancer Institute \& Research Centre, Delhi. Eligibility criteria included 1) Histologically proven squamous cell carcinomas of oral cavity, oropharynx, hypopharynx, 
2) Age 18-70 years, 3) Stage III-IVb disease (AJCC 7 th edition), 4) ECOG performance score 0-2 and 5) Informed consent taken. Study was approved by the Ethics Committee of the hospital.

\section{Treatment planning}

All patients underwent immobilisation using thermoplastic cast, CT simulation, and treatment planning according to the departmental protocol. Planning CT scans were acquired with $3 \mathrm{~mm}$ axial images from glabella to tracheal bifurcation and images were transferred to Eclipse contouring station. TV and OARs were contoured as per institutional protocol.

GTV represented gross visible tumor and/or enlarged lymphnodes identified clinically or on CT. CTV included regions at high risk of microscopic disease and a $0.5-2 \mathrm{~cm}$ margin on GTV was given. Asymmetric PTV margin of $2-5 \mathrm{~mm}$ was used to account for daily setup errors. Dose prescription of 70Gy to GTV, 66Gy to CTV and 60Gy to PTV was given.

IMRT plans were generated and approved for each patient using Eclipse Treatment Planning System (Version 11.0 Varian Medical System, Palo Alto, California, USA). All treatments were delivered by using Varian True Beam linear accelerator. Original treatment plan (OPLAN) was implemented for whole course of radiotherapy. Treatment was delivered once daily, 5 days/week, over 7 weeks.

\section{Adaptive radiotherapy: $C T$ re-simulation and IMRT replanning}

Clinically observed changes in patient's anatomy or weight loss were identified during the course of treatment and a second planning scan was acquired for each patient at a delivered dose of $40 \mathrm{~Gy}$ (95\% GTV). New cast was made for immobilization in case of significant change in patient contour. TV and OARs were manually recontoured and their volumes recorded. OPLAN was delivered for entire course of treatment.

Second CT scan acquired after 4 weeks was used to generate a new IMRT replan (RPLAN) (To see dosimetric changes on replanning). Hybrid IMRT plan (HPLAN) represented a situation in which no replanning would have occurred. HPLAN applied beam configuration of OPLAN (including intensity profile of each beam) to the anatomy of second scan.

For positional analysis, centre of mass (COM) was determined from CT scan with respect to a reference point ie fiducial marker (At which DICOM centre was set) and this point was used as a common reference for evaluation of positional shifts of parotids.

Anatomical changes observed after 4 weeks (20\#) of radiotherapy were compared between the 2 scans. For each IMRT plan, dose distribution to TVs and OARs was generated. IMRT plan (OPLAN30 - Based on first CT scan for remaining 30Gy) was compared to HPLAN30 (Hybrid plan for 30Gy) to ensure dosimetric consistency between two plans. To investigate effect of replanning on dosimetric outcome, HPLAN30 was compared with the IMRT replan (RPLAN30). This comparison accounted for remaining fractions to be delivered during second half of treatment.

\section{Outcome measures}

Dosimetric comparison for GTV, CTV, PTV, parotids and spinal cord was performed for each of the 3 plans. Data was analysed by applying the standard statistical tests using SPSS Statistics 17.0; SPSS Inc., Chicago, IL, USA. Paired t-test was used if the distribution of difference between groups was normally distributed. Otherwise, non-parametric Wilcoxon signed-rank test was used for comparison of means between each plan. A p-value $<0.05$ was considered statistically significant. Dosimetric differences between various plans were correlated with absolute and relative GTV or parotid gland shrinkage, parotid gland (COM) shift \& weight loss. Because of small sample size, results were confirmed with Spearman's correlation.

\section{Results}

Thirty patients with LAHNC were analysed for dosimetric and volumetric changes occurring during course of IMRT (Figure 1-2). Patient characteristics are summarized in Table 1. All patients underwent IMRT alongwith concurrent weekly chemotherapy with Injection
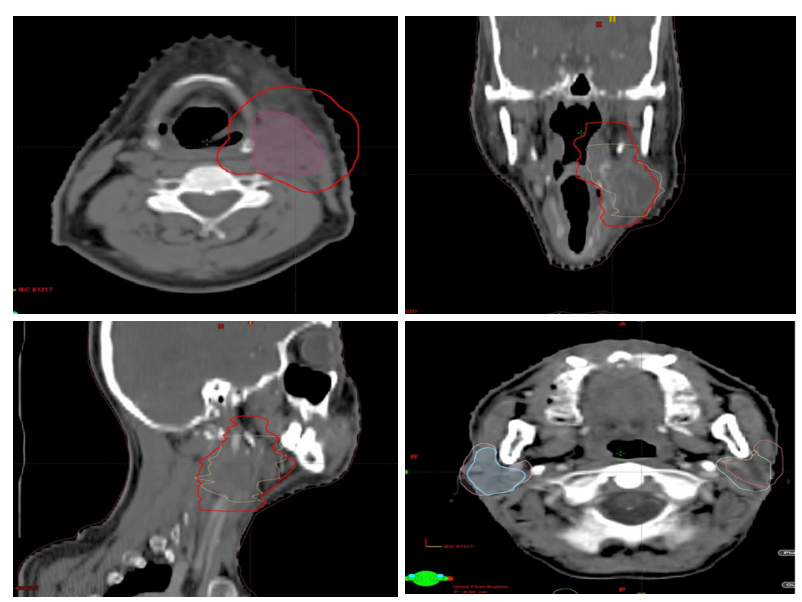

Figure 1. Replanning CT Scan Images (acquired after 4 weeks of treatment). Volumetric change in gross tumor volume is shown in a) axial, b) coronal and c) sagittal sections. Volumetric and positional changes in ipsilateral and contralateral parotids are visible in panel d)

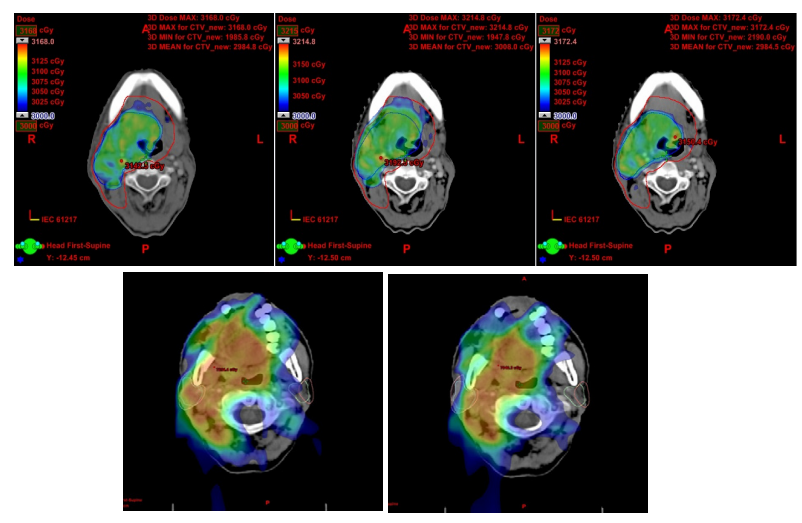

Figure 2. Comparison of Dose Distribution of Target Volume. a) Initial treatment plan, b) Hybrid plan and c) Replan. Dosimetric comparison of parotids in hybrid plan and replan is shown in panel d) and e) 
Table 1. Patient Characterstics

\begin{tabular}{lc}
\hline \multicolumn{1}{c}{ Characterstics } & $\mathrm{N}=30$ \\
\hline Age (Mean+SD) & $54.90+9.40$ years \\
Sex (M: F) & $26(86.67 \%): 4(13.33 \%)$ \\
ECOG Performance score & \\
1:2:3:4 & $26(86.67 \%): 4(13.33 \%): 0(0 \%): 0(0 \%)$ \\
Smoker & $20(66.67 \%): 10(33.33 \%)$ \\
Yes:No & $14(46.66 \%): 16(53.34 \%)$ \\
Alcohol & $10(33.33 \%)$ \\
Yes:No & $15(50 \%)$ \\
Site & $05(16.67 \%)$ \\
Oral Cavity & \\
Oropharynx & \\
Hypopharynx & \\
Clinical T-stage & \\
T1:T2:T3:T4 & $0(0 \%): 6(20 \%): 11(36.67 \%): 13(43.33 \%)$ \\
N-stage & \\
N1:N2a:N2b:N2c:N3 & $0(0 \%): 0(0 \%): 6(20 \%): 21(70 \%): 3(10 \%)$ \\
\hline
\end{tabular}

Cisplatin.

\section{Volumetric Analysis}

Table 2 compared volumes of TV and OARs between 2 time intervals. Mean shrinkage of TV (GTV/CTV/PTV) and OARs (Parotids) after 4 weeks of radiotherapy was statistically significant $(\mathrm{p}<0.05)$.

\section{Dosimetric analysis}

Impact of anatomical changes in TV and OARs on dosimetric outcome was evaluated. A summary of dosimetric characteristics of TVs are summarized in Table 3. With regards to GTV, no significant difference in TV coverage (D98\%/D95\%) on comparing OPLAN30 vs HPLAN30 ( $\mathrm{p}=0.478)$ and HPLAN30 vs RPLAN30 $(\mathrm{p}=0.681)$ was noted. However, replanning induced a significant difference in D2\% as compared to HPLAN30 for GTV. On comparing planned and delivered dose, a substantial increase in $\mathrm{V}>110 \%$ was seen $(\mathrm{p}=0.048)$ in HPLAN30 and adaptive planning led to its reduction $(\mathrm{p}=0.001) . \mathrm{V}<93 \%$ of HPLAN30 $(0.14+0.47 \%)$ was higher in comparison to OPLAN30 and RPLAN30

Table 2. Evaluation of Volume Parameters during the Course of Treatment

\begin{tabular}{lccc}
\hline & $\begin{array}{c}\text { Pre-treatment volume } \\
(\mathrm{cc})(\text { Mean+SD) }\end{array}$ & $\begin{array}{c}\text { Mid treatment volume } \\
(\mathrm{cc})\left(\begin{array}{c}\text { (After 4 weeks of } \\
\text { treatment) }\end{array}\right.\end{array}$ & $\begin{array}{c}\text { Absolute change in } \\
\text { volume (cc) }\end{array}$ \\
\cline { 3 - 4 } (Mean+SD) & $\begin{array}{c}\text { Mean percentage } \\
\text { change in volume }(\%)\end{array}$ \\
\hline GTV & $79.34+34.84$ & $44.54+29.47$ & $34.80+16.60$ \\
PTV & $127.84+49.86$ & $78.04+48.49$ & $49.80+21.00$ \\
I/L PAROTID & $185.61+58.70$ & $117.13+66.58$ & $68.47+38.22$ \\
C/L PAROTID & $17.64+7.62$ & $11.54+5.38$ & $6.09+3.56$ \\
\hline
\end{tabular}

Table 3. Dosimetric Evaluation of the Target Volumes for Original Plan, Hybrid Plan and Re-plan

\begin{tabular}{|c|c|c|c|c|c|}
\hline & OPLAN30 & HPLAN30 & RPLAN30 & $\begin{array}{c}\text { HPAN30 vs } \\
\text { OPLAN30 (p-value) }\end{array}$ & $\begin{array}{c}\text { HPAN30 vs RPLAN30 } \\
\text { (p-value) }\end{array}$ \\
\hline D98\% (Gy) & $29.66+0.65$ & $29.57+1.13$ & $29.54+0.7$ & 0.478 & 0.681 \\
\hline D95\% (Gy) & $30.04+0.55$ & $29.97+0.97$ & $29.7+0.58$ & 0.526 & 0.24 \\
\hline D50\% (Gy) & $31.29+0.63$ & $31.37+0.96$ & $30.66+0.48$ & 0.117 & 0.004 \\
\hline $\mathrm{D} 2 \%$ (Gy) & $32.78+0.90$ & $33.10+1.60$ & $31.49+0.6$ & 0.191 & $<0.01$ \\
\hline $\mathrm{V}>110 \%(\%)$ & $2.72+4.47$ & $9.52+13.47$ & $0.55+2.12$ & 0.048 & 0.001 \\
\hline $\mathrm{V}<93 \%(\%)$ & $0.08+0.15$ & $0.14+0.47$ & $0.07+0.22$ & 0.767 & 0.866 \\
\hline \multicolumn{6}{|l|}{ CTV } \\
\hline D98\% (Gy) & $28.58+1.42$ & $28.38+2.58$ & $28.44+0.92$ & 0.794 & 0.654 \\
\hline D95\% (Gy) & $29.38+1.25$ & $29.24+1.76$ & $28.96+0.75$ & 0.263 & 0.156 \\
\hline D50\% (Gy) & $31.12+0.87$ & $31.26+1.08$ & $30.23+0.51$ & 0.117 & 0.001 \\
\hline D2\% (Gy) & $32.77+0.97$ & $33.31+2.05$ & $31.42+0.56$ & 0.086 & $<0.01$ \\
\hline $\mathrm{V}>110 \%(\%)$ & $14.28+23.40$ & $22.72+26.07$ & $8.11+14.77$ & 0.04 & 0.003 \\
\hline $\mathrm{V}<93 \%(\%)$ & $0.22+0.37$ & $0.76+0.98$ & $0.10+0.19$ & 0.04 & 0.002 \\
\hline \multicolumn{6}{|l|}{ PTV } \\
\hline D98\% (Gy) & $27.95+1.43$ & $27.15+3.23$ & $27.33+1.07$ & 0.526 & 0.765 \\
\hline D95\% (Gy) & $28.68+1.29$ & $28.36+2.26$ & $27.85+0.89$ & 0.97 & 0.145 \\
\hline D50\% (Gy) & $30.83+1.10$ & $31.07+1.21$ & $32.78+1.06$ & 0.086 & 0.001 \\
\hline D2\% (Gy) & $32.78+1.06$ & $33.31+2.23$ & $31.37+0.53$ & 0.156 & $<0.01$ \\
\hline $\mathrm{V}>110 \%(\%)$ & $27.87+28$ & $39.53+21.79$ & $18.09+21.79$ & 0.433 & 0.02 \\
\hline $\mathrm{V}<93 \%(\%)$ & $0.42+0.48$ & $0.92+1.2$ & $0.22+0.30$ & 0.5 & 0.049 \\
\hline
\end{tabular}


Table 4. Dosimetric Evaluation of Spinal cord and Parotids

\begin{tabular}{|c|c|c|c|c|c|}
\hline \multicolumn{6}{|c|}{ Spinal cord $(\mathrm{Gy})$} \\
\hline & OPLAN30 & HPLAN30 & RPLAN30 & $\begin{array}{c}\text { HPAN30 vs } \\
\text { OPLAN30 (p-value) }\end{array}$ & $\begin{array}{c}\text { HPAN30 vs RPLAN30 } \\
\text { (p-value) }\end{array}$ \\
\hline Dmax & $17.62+2.36$ & $20.09+1.34$ & $14.34+1.72$ & $<0.01$ & $<0.01$ \\
\hline $\mathrm{D} 2 \%$ & $16.95+1.40$ & $18.58+1.75$ & $12.70+1.74$ & 0.001 & $<0.01$ \\
\hline $\mathrm{D} 1 \%$ & $17.42+1.39$ & $19.45+1.93$ & $13.25+2.04$ & 0.001 & $<0.01$ \\
\hline \multicolumn{6}{|c|}{ Ipsilateral Parotid (Gy) } \\
\hline Dmean & $15.15+4.19$ & $18.45+6.84$ & $12.81+5.16$ & 0.01 & 0.001 \\
\hline D66\% & $10.05+5.30$ & $13.65+8.78$ & $9.13+5.43$ & 0.05 & 0.019 \\
\hline D50\% & $14.03+5.56$ & $17.77+8.21$ & $11.41+5.87$ & 0.028 & 0.002 \\
\hline D33\% & $18.84+5.05$ & $22.45+6.92$ & $14.55+6.47$ & 0.02 & 0.001 \\
\hline \multicolumn{6}{|c|}{ Contralateral Parotid (Gy) } \\
\hline Dmean & $11.79+3.00$ & $14.01+5.19$ & $11.77+4.41$ & 0.08 & 0.07 \\
\hline D66\% & $6.65+2.78$ & $9.15+6.37$ & $8.09+5.08$ & 0.09 & 0.76 \\
\hline D50\% & $9.90+4.13$ & $12.60+7.21$ & $10.49+4.87$ & 0.17 & 0.45 \\
\hline D33\% & $14.37+4.87$ & $16.84+6.96$ & $13.73+5.30$ & 0.16 & 0.02 \\
\hline
\end{tabular}

$(\mathrm{p}<0.05)$. HI was worse for HPLAN30 $(0.11+0.04)$ as compared to OPLAN30 $(0.05+0.02)$ and RPLAN30 $(0.06+0.029)$. With replanning, dose distribution to GTV was made more homogenous $(\mathrm{p}<0.01)$. Similar dosimetric outcome was observed for CTV and PTV. Adaptive replanning provided more uniform tumor coverage as compared to the hybrid plan.

Table 4 showed increase in dose to OARs without replanning after 4 weeks of treatment. A statistically significant increase in Dmax, D2\% and D1\% was noted for spinal cord in HPLAN30 when compared to OPLAN30. Replanning reduced mean Dmax, D2\% and D1\% by $28.26+10.27 \%, 30.87+12.83 \%$ and $31.20+13.09 \%$ respectively as compared to delivered dose $(\mathrm{p}<0.01)$. Mean dose, D66\%, D50\% and D33\% to ipsilateral parotid were significantly higher in HPLAN30 as compared to OPLAN30 ( $\mathrm{p}=0.01)$. Mean dose to ipsilateral parotid was significantly reduced with replanning by $26.04+29.14 \%$ $(\mathrm{p}=0.001)$. Other parameters (D33\%, D50\% and D66\%) also reduced considerably after replanning $(\mathrm{p}<0.05)$. Similar pattern was obtained for contralateral parotid; however the difference was not significant.

During the course of radiotherapy, COM of ipsilateral parotid shifted medially by mean value of $0.33+0.34 \mathrm{~cm}$ in $\mathrm{X}$-axis, $0.13+0.74 \mathrm{~cm}$ in $\mathrm{Y}$-axis and by $-0.25+0.93 \mathrm{~cm}$ in Z-axis. Mean shift in contralateral parotid was $0.27+0.23 \mathrm{~cm},-0.03+0.69 \mathrm{~cm}$ and $-0.27+0.78 \mathrm{~cm}$ in $\mathrm{X}$, $\mathrm{Y}$ and $\mathrm{Z}$-axis respectively. Total average weight loss of $4.62+2.42 \%$ between weeks $0-4$ of treatment (Total weight loss during entire treatment $8.45+2.70 \%$ ) was recorded, with an average weekly loss being $0.7+0.32 \%$. Percentage weight loss between weeks 0-4 significantly correlated with percentage change in ipsilateral parotid volume (Correlation coefficient 0.48, $\mathrm{p}=0.03$ ). No significant correlation between weight loss after 4 weeks, GTV shrinkage and parotid shift in $\mathrm{X}, \mathrm{Y}$ and $\mathrm{Z}$ axis was seen. Pre-treatment GTV volume and absolute GTV shrinkage was found to significantly impact ipsilateral parotid dose (relative and absolute, $\mathrm{p}<0.05$ ).

\section{Discussion}

IMRT generates steep dose gradients around the TV; therefore it is important to be aware of the dosimetric impact occurring due to anatomical/positional variations. These changes might result in underdosage of TV and higher than tolerance doses to OARs. In the present study, patients were evaluated for (1) Dosimetric and volumetric changes in TV's and OARs and (2) To study effect of replanning on dose distribution.

Radiotherapy for HNC is often associated with weight loss. Barker et al. (2004) and Ho et al. (2012) reported a median weight loss of $7.1 \%$ and $7.6 \%$ respectively during entire course of treatment. Some authors noticed considerable weight loss after 3rd week of radiotherapy (Bhandari et al., 2014). In accordance with above studies, mean weight loss in our study was $4.62 \%$ in 1 st 4 weeks of treatment with 8 patients requiring nasogastric tube and 3 requiring percutaneous endoscopic gastrostomy tube insertions.

Anatomical Modifications: Volume of TV and OARs were compared among scans at two intervals. Many patients showed marked anatomical changes during course of their treatment. These included shrinkage of primary tumor and nodal volumes, edema or weight loss (Barker et al., 2004; Bhinde et al., 2010; Hansen et al., 2006; Castadot et al., 2010).

Target volume reduction during radiotherapy for $\mathrm{HNC}$ has been observed by many studies. Cheng et al. (2012) documented significant decrease in GTV in HPLAN in comparison to OPLAN. Barker et al. (2004) obtained CT images during treatment thrice a week and quantified volumetric changes during this period. They noted GTV regression of approximately $70 \%$ during entire treatment at $1.8 \%$ /day and this regression tended to be asymmetrical.

Geets et al. (2007) assessed modification in TV and dose distribution during chemoradiation for 10 patients with LAHNC and found significant reduction in GTV leading to corresponding decrease in CTV/PTV. Vasquez Osorio et al. (2008) performed re-simulation at 46Gy and observed decrease by $25 \pm 15 \%$ in primary tumor volume. In the present study, TV contours were compared 
between 2 scans and a significant reduction in mean GTV $(34.80+16.60 \mathrm{cc}, 47.62+20.85 \%, \mathrm{p}<0.01)$ was seen that was similar to tumor reductions reported in previous studies (Barker et al., 2004; Bhinde et al., 2010; Hansen et al., 2006). In accordance with study by Cheng et al. (2012), no correlation was found between weight loss and volumetric changes in GTV. Weight loss thus cannot always predict for volumetric changes in TV, probably due to other confounding factors.

Volumetric and positional changes were not confined to TV only. Studies have observed significant volume loss with medial displacement of parotids during radiotherapy (Barker et al., 2004). In the present study, mean volume of ipsilateral and contralateral parotids decreased during course of treatment (after 4 weeks) (Median \% decrease: $32.9 \%$ for ipsilateral, $34.10 \%$ for contralateral parotid).

Parotid volume contraction in our study was in agreement with published results (Brouwer et al., 2015; Hansen et al., 2006; Ho et al., 2012; Wang W et al., 2010). Brouwer et al. (2015) summarized specific changes occurring during radiotherapy and noted an average volume decrease in parotids of $26+11 \%$. Ho et al. (2012) noted mean reduction in volume of ipsilateral and contralateral parotid by $29.7 \%$ and $28.4 \%$ respectively. Wang et al. (2010) documented mean reduction of $20.6 \%$ and $19.8 \%$ in left and right parotid volume respectively, while Hansen et al. (2006) reported a volume reduction of $21.5 \%$ and $15.6 \%$ in left and right parotids. In accordance with findings of Schwartz et al. (2012), a significant correlation was observed between weight loss and percent reduction in ipsilateral parotid volume in present study.

Weight loss may shift parotids to high dose regions, thereby resulting in parotid shrinkage. COM of parotids on both sides shifted medially over time. Median medial shift was $0.19 \mathrm{~cm}$ for ipsilateral and $0.20 \mathrm{~cm}$ for contralateral parotids. Lee C et al. (2008) and Nishi et al. (2013) reported a medial parotid shift of $5.26 \mathrm{~mm}$ and $4.2 \mathrm{~mm}$ respectively. Barker et al. (2004) observed a significant correlation between medial displacements of parotid gland with ongoing weight loss, thereby indicating weight loss as a surrogate for parotid shift. In contrast to above finding, no such correlation was documented in our study; thereby demonstrating its unpredictive nature. To quantify the amount of shift, CT scan during therapy may thus become necessary.

Dosimetric Impact of Anatomical Modifications: Due to alteration in patient's anatomy, the actual delivered dose may not correspond to the planned dose and have an adverse effect on treatment outcome in terms of tumor control and normal tissue complications. Barker et al. (2004) reported significant underdosing of PTV or overdosing of parotids if these alterations were not accounted for with replanning.

Target Volumes (Planned vs Delivered dose): In present study, dose comparisons between "planned", "hybrid/delivered" and "adaptive" plans was performed. Doses actually delivered to TVs (as derived from HPLAN30) didn't significantly differ from planned doses with a significant increase in $\mathrm{V}>110 \%$ observed in HPLAN30. Their findings were in line with previous studies (Castadot et al., 2010; O'Daniel JC et al., 2007;
Wu Q et al., 2009) showing no change in TV coverage with shrinkage.

However, some studies have shown contradictory results and demonstrated compromised TV coverage (Hansen et al., 2006; Cheng et al., 2012; Wang W et al., 2010). Cheng et al. (2012) observed increase in radiation dose to TVs which was due to weight loss. Hansen et al. (2006) and Bhandari et al. (2014) compared dosimetric effects of replanning versus no replanning and observed significant decrease in TV coverage without replanning.

Dosimetric Changes in OARs: Dose to various OARs increased during radiotherapy in the present study.

Spinal cord: There was a significant increase in delivered dose to spinal cord when compared to planned dose. Cheng et al. (2012) reported a significantly higher mean Dmax in HPLAN as compared to OPLAN ( $\mathrm{p}=0.008)$ after both 30Gy and 50Gy. Hansen et al. (2006) indicated rise in maximum dose delivered to cord in all cases without replanning. Castadot et al. (2010) and Han C et al. (2008) documented increase in actual dose delivered to spinal cord as compared to planned dose.

Ipsilateral parotid: Mean dose increased significantly by $21.7 \%$ in HPLAN30 ( $p=0.007)$ as compared to OPLAN30. Medial displacement of parotids due to tumor regression and weight loss tends to bring parotids into higher isodose volume, thereby resulting in larger parotid dose than anticipated (Blanco AI et al., 2005).

In a study comparable to present one, Wu Q et al. (2009) and Lee et al. (2008) demonstrated increase in cumulative Dmean by $10 \%$ for parotids. Cheng et al. (2012) reported increase in Dmedian of contralateral and ipsilateral parotids by 6.9 and $24.1 \%$ respectively after 30 Gy of radiation. O'Daniel et al. (2007) anticipated increase in median ipsilateral parotid dose by $3 \mathrm{~Gy}$, and concluded that dose delivered to parotids was 5-7Gy higher than planned dose in $45 \%$ patients. Blanco et al. (2005) showed decrease in salivary function at a rate of $5 \%$ with increase in mean dose by 1Gy. Castelli et al. (2015) observed parotid overdosing in $59 \%$ parotids with an average increase of 3.7Gy without replanning. Han et al. (2008) noted greater median dose in later phase of treatment when repeat CT scan done was used to recalculate dosimetry.

Factors contributing to discrepancy between planned and delivered doses include weight loss, tumor shrinkage and treatment positioning errors. Anatomical changes during treatment are most probable explanation for higher dose delivered to OARs (Castadot et al., 2011). Castadot et al. (2010) noted pre-treatment GTV volume and GTV shrinkage to be suitable indicators of additional dose delivered to cord and parotids. Similar correlation was observed in our study with regards to parotid dose $(\mathrm{p}<0.05)$. Thus, tumor burden and its contraction during treatment appear to be significant parameters for selecting patients who may benefit from adaptive strategy for preserving parotid functioning.

Ho et al. (2012) attributed weight loss as being a contributing factor for difference between planned and delivered doses to parotid. In contrast to findings of Ho et al. (2012) and Barker et al. (2004), analysis in our study did not demonstrate any correlation between weight loss and its effects on parotid dose distribution. O'Daniel 
et al. (2007) recognized parotid dose changes to be a consequence of parotid shift. However our study didn't reveal any such correlation.

Several studies have reported that application of original plan to altered anatomy leads to higher dose than planned to OARs (Hansen et al., 2006; Castadot et al., 2012; O'Daniel et al., 2007). This result raised the question of adaptive replanning. Various investigators have studied replanning during radiation and concluded that replanning could counterbalance for dosimetric degradation occurring due to anatomical changes (Hansen et al., 2006; Mohan R et al., 2005).

Significant difference was observed between delivered and adaptive doses to parotid. Our initial analysis confirmed that mean dose to ipsilateral parotid improved with ART by $26.04+29.14 \%$. This is in line with findings of Wu Q et al. (2009), who concluded that a single midcourse ART replanning could provide parotid sparing of similar magnitude. Strategies like replanning or reducing margins help achieve upto $30 \%$ decrease in parotid dose of which majority (2/3rd) is from margin reduction (Wu et al., 2009). Schwartz et al. (2012) reported mean parotid dose sparing by $3.9 \%$ and $3.8 \%$ in contralateral parotid, and by $2.8 \%$ and $9 \%$ in ipsilateral parotid with single and two ART planning respectively. Castadot et al. (2011) noted reduction in mean parotid dose after one, two and six replanning by $3 \%, 5 \%$ and $6 \%$ respectively.

Kuo et al. (2006) conducted a prospective trial of 10 patients replanned after $45 \mathrm{~Gy}$ and showed reduction in parotid dose by approximately $2-4 \mathrm{~Gy}$ with replanning. In another series of 33 patients, repeat $\mathrm{CT}$ and planning were performed (Zhao et al., 2011). They reported an increase in dose to normal structures in hybrid plan. Repeat CT imaging and IMRT replanning was thus recommended with a larger potential benefit in preservation of parotid functioning.

In the spinal cord, actual delivered dose deviated significantly from the planned dose. Dmax, D2\% and D1\% could be reduced by $28.26+10.27 \%, 30.87+12.83 \%$ and $31.20+13.09 \%$ using re-planning. Castadot et al. (2011) and Hansen et al. (2006) reported significant improvement in dose distribution for spinal cord with adaptive strategy.

For mid-course replanning, adaptive isodose volumes receiving $>110 \%$ and $<93 \%$ of prescribed dose were significantly lesser than delivered doses in present study. Adaptive replanning did not induce any significant difference in D98\%/D95\% as compared to delivered dose. HI of TV was worse in hybrid plan, indicating that dose distribution can be made more homogenous with re-planning. Thus, above findings indicate better TV coverage with application of the adaptive plan.

Hansen et al. (2006) noted improvement in D95\% and V93\% for PTV. Simon et al. (2011) observed comparable TV coverage with reduction in doses to OARs after replanning. Wu Q et al. (2009) reported minimal effect on TVs coverage with replanning. Jensen et al. (2012) reported improvement in target coverage by upto $10.7 \%$ (median dose) with replanning.

Regarding timing of replanning, a crucial challenge at present is to decide as to when and how frequently to replan, as there are no clear consensus guidelines available. Adaptive strategy is unlikely to benefit all patients and a criterion is required to identify such patients. Different authors have reported varying time intervals for rescanning. Anatomical changes are more pronounced in first half of treatment, especially after second week of treatment (Barker et al., 2004; Bhinde et al., 2010; Wang et al., 2009; Sanguineti et al., 2013; Hunter et al., 2013). Bhandari et al. (2014) and Schwartz et al. (2012) observed maximal anatomical changes between 3rd and 4th week. Yang et al. (2011) demonstrated 4-5th week of treatment as ideal time for assessing response, while keeping sufficient time for replanning. Therefore, the most appropriate time for reassessment and replanning might be $>2$ weeks after beginning radiotherapy. Adaptive replanning done in later phase of radiation might limit its advantage due to inadequate response time.

Wu et al. (2009) concluded that planning >once a week was unnecessary and that greatest benefit of replanning was seen when performed at week 4 of treatment. Wang W et al. (2012) highlighted significance of replanning before 25th fraction during IMRT. Cheng et al. (2012) recommended replanning with repeat CT scan at $30 \mathrm{~Gy}$ to keep dose to TV satisfactory. Schwartz DLet al. (2012) suggested one or two mid-treatment replanning. Woodford $\mathrm{C}$ et al. (2007) recommended adaptive replanning if GTV decreased by $>30 \%$ at any point during first 20 fractions. Bhandari et al. (2014) recommended repeat scanning and replanning for patients with body mass index $(\mathrm{BMI})>25 \mathrm{~kg} / \mathrm{m} 2$, as greater reduction in body weight and consequently TV was noted for patients with higher BMI during chemoradiation. Beltran M et al. (2012) suggested body weight to be a dependent factor to detect changes in irradiated body contours. Another study suggested significant weight loss, ill-fitting cast and GTV shrinkage to be markers for replanning (Chen et al., 2014).

Daily replanning is not feasible considering the limited reserve and increased time and effort required, thereby leading to substantial burden on physicist, physician and institute (Berwouts D et al., 2013; Budach W et al., 2011; Daisne JF et al., 2013). It may thus be more practical to replan once or twice during treatment.

Major drawback of our study was small number of patients; as a result clinical outcome of adaptive strategies during radiotherapy was not evaluated. Some tool may be required to identify patients who may benefit from mid-treatment replanning, thereby reducing workload and increasing cost effectiveness. Our study demonstrated that use of one or two repeat imaging and adaptive planning provides greater normal tissue sparing alongwith improved TV coverage.

\section{References}

Barker JL Jr, Garden AS, Ang KK, et al (2004). Quantification of volumetric and geometric changes occurring during fractionated radiotherapy for head-and- neck cancer using an integrated CT/linear accelerator system. Int J Radiat Oncol Biol Phys, 59, 960-70.

Beltran M, Ramos M, Rovira JJ, et al (2012). Dose variations in tumor volumes and organs at risk during IMRT for headand-neck cancer. J Appl Clin Med Phys, 13, 3723. 
Berwouts D, Olteanu LA, Duprez F, et al (2013). Three-phase adaptive dose-painting-by-numbers for head-and-neck cancer: initial results of the phase I clinical trial. Radiother Oncol, 107, 310-6.

Bhandari V, Patel P, Gurjar OP, et al (2014). Impact of repeat computerized tomography replans in the radiation therapy of head and neck cancers. J Med Phys, 39, 164-8.

Bhinde SA, Davies M, Burke K, et al (2010). Weekly volumetric and dosimetric changes during chemoradiotherapy with IMRT for head and neck tumours: A prospective observational study. Int J Radiat Oncol Biol Phys, 76, $1360-8$.

Blanco AI, Chao KSC, El Naqa I, et al (2005). Dose-volume modelling of salivary function in patients with head-andneck cancer receiving radiotherapy. Int J Radiat Oncol Biol Phys, 62, 1055-69.

Brouwer CL, Steenbakkers RJ, Langendijik JA, et al (2015). Identifying patients who may benefit from adaptive radiotherapy: Does the literature on anatomic and dosimetric changes in head and neck organs at risk during radiotherapy provide information to help? Radiother Oncol, 115, 285-94.

Budach W, Bolke E, Fietkau R, et al (2011). Evaluation of time, attendance of medical staff, and resources during radiotherapy for head and neck cancer patients: the DEGROQUIRO trial. Strahlenther Onkol, 187, 449-60.

Castadot P, Lee JA, Geets X, et al (2010). Adaptive Radiotherapy of Head and Neck Cancer. Semin Radiat Oncol, 20, 84-93.

Castadot P, Geets X, Lee JA, et al (2011). Adaptive functional image-guided IMRT in pharyngo-laryngeal squamous cell carcinoma: Is the gain in dose distribution worth the effort? Radiother Oncol, 101, 343-50.

Castelli J, Simon A, Louvel G, et al (2015). Impact of head and neck cancer adaptive radiotherapy to spare the parotid glands and decrease the risk of xerostomia. Radiother Oncol, 10, 6 .

Charles B. Simone II a, David Ly, et al (2011). Comparison of intensity-modulated radiotherapy, adaptive radiotherapy, proton radiotherapy, and adaptive proton radiotherapy for treatment of locally advanced head and neck cancer, Radiother Oncol, 101, 376-82.

Chen AM, Daly ME, Cui J, et al (2014). Clinical outcomes among patients with head and neck cancer treated by intensity-modulated radiotherapy with and without adaptive replanning. Head Neck, 36, 1541-6.

Cheng HCY, Vincent W.C, Roger K.C, et al (2012). A prospective study on volumetric and dosimetric changes during intensitymodulated radiotherapy for nasopharyngeal carcinoma patients. Radiother Oncol, 104, 317-23.

Daisne JF, Blumhofer A (2013). Atlas-based automatic segmentation of head and neck organs at risk and nodal target volumes: a clinical validation. Radiother Oncol, 8, 154 .

Geets X, Tomsej M, Lee JA, et al (2007). Adaptive biological image-guided IMRT with anatomic and functional imaging in pharyngo-laryngeal tumors. Impact on target volume delineation and dose distribution using helical tomotherapy. Radiother Oncol, 85, 105-15.

Han C, Chen YJ, Liu A, et al (2008). Actual dose variation of parotid glands and spinal cord for nasopharyngeal cancer patients during radiotherapy. Int J Radiat Oncol Biol Phys, 70, 1256-62.

Hansen EK, Bucci MK, Quivey JM, et al (2006). Repeat CT imaging and replanning during the course of IMRT for headand-neck cancer. Int J Radiat Oncol Biol Phys, 64, 355-62.

Ho KF, Moore C, Webster G (2012). Monitoring dosimetric impact of weight loss with kilovoltage $(\mathrm{KV})$ cone beam CT (CBCT) during parotid-sparing IMRT and concurrent chemotherapy. Int J Radiation Oncol Biol Phys, 82, 375-82.

Hunter KU, Fernandes LL, Vineberg KA (2013). Parotid glands dose-effect relationships based on their actually delivered doses: implications for adaptive replanning in radiation therapy of head-and-neck cancer. Int J Radiat Oncol Biol Phys, 87, 676-82.

Jensen AD, Nill S, Huber PE, et al (2012).Clinical concept for interfractional adaptive radiation therapy in the treatment of head-and-neck cancer. Int J Radiat Oncol Biol Phys, 82, 590-6.

Kuo YC, Wu TH, Chung TS, et al (2006). Effect of regression of enlarged neck lymph nodes on radiation doses received by parotid glands during intensity-modulated radiotherapy for head and neck cancer. Am J Clin Oncol, 29, 600-5.

Lee C, Langen KM, Lu W, et al (2008). Assessment of parotid gland dose changes during head and neck cancer radiotherapy using daily megavoltage computed tomography and deformable image registration. Int J Radiat Oncol Biol Phys, 71, 1563-71.

Mohan R, Zhang X, Wang H, et al (2005). Use of deformed intensity distributions for on-line modification of imageguided IMRT to account for interfractional anatomic changes. Int J Radiat Oncol Biol Phys, 61, 1258-66.

Nath SK, Simpson DR, Rose BS, et al (2009). Recent advances in image-guided radiotherapy for head and neck carcinoma. J Oncol, 2009.

Nishi T, Nishimura Y, Shibata T, et al (2013). Volume and dosimetric changes and initial clinical experience of a two-step adaptive intensity modulated radiation therapy (IMRT) scheme for head and neck cancer. Radiother Oncol, 106, 85-9.

O'Daniel JC, Garden AS, Schwartz DL, et al (2007). Parotid gland dose in intensity-modulated radiotherapy for head and neck cancer: Is what you plan what you get? Int J Radiat Oncol Biol Phys, 69, 1290-93.

Sanguineti G, Ricchetti F, Thomas O, et al (2013). Pattern and predictors of volumetric change of parotid glands during intensity modulated radiotherapy. Br J Radiol, 86, 20130363.

Schwartz DL, Garden AS, Thomas J et al (2012). Adaptive Radiotherapy for Head-and-Neck Cancer: Initial Clinical Outcomes from a Prospective Trial. Int J Radiation Oncol Biol Phys, 83, 986-93.

Vasquez Osorio EM,Hoogeman MS, Al-Mamgani A, et al (2008). Local anatomic changes in parotid and submandibular glands during radiotherapy for oropharynx cancer and correlation with dose, studied in detail with nonrigid registration. Int $J$ Radiat Oncol Biol Phys, 70, 875-82.

Wang W, Yang H, Hu W et al (2010). Clinical study of the necessity of replanning before the 25th fraction during the course of intensity-modulated radiotherapy for patients with nasopharyngeal carcinoma. Int J Radiat Oncol Biol Phys, 77, 617-21.

Wang ZH, Yan C, Zhang ZY, et al (2009). Radiation-induced volume changes in parotid and submandibular glands in patients with head and neck cancer receiving postoperative radiotherapy: a longitudinal study. Laryngoscope, 119, 1966-74.

Woodford C, Yartsev S, Dar R Bauman G (2007). Adaptive radiotherapy planning on decreasing gross tumor volumes as seen on megavoltage computed tomography images. Int J Radiat Oncol Biol Phys, 69, 1316-22.

Wu Q, Chi Y, Chen PY, et al (2009). Adaptive replanning strategies accounting for shrinkage in head and neck IMRT. Int J Radiat Oncol Biol Phys, 75, 924-32.

Yan D, Lockman D (2001). Organ/patient geometric variation in external beam radiotherapy and its effect. Med Phys, 28, 593-602.

Yang SN, Liao CY, Chen SW, et al (2011). Clinical implications of the tumor volume reduction rate in head-and-neck cancer 
Abhinav Dewan et al

during definitive intensity-modulated radiotherapy for organ preservation. Int J Radiat Oncol Biol Phys, 79, 1096-103.

Zhao L, Wan Q, Zhou Y, et al (2011). The role of replanning in fractionated intensity modulated radiotherapy for nasopharyngeal carcinoma. Radiother Oncol, 98, 23-7. 